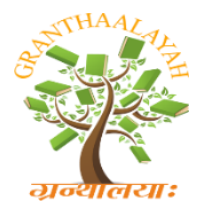

$$
\begin{gathered}
\text { INTERNATIONAL JOURNAL OF RESEARCH } \\
\text { GRANTHAALAYAH } \\
\text { A knowledge Repository }
\end{gathered}
$$

Science

\title{
GRAVITY, PROBABILITY AND CONSCIOUSNESS
}

\author{
Prashant ${ }^{* 1}$ \\ ${ }^{* 1}$ Tata Consultancy Services, Mumbai Maharashtra-400606, India
}

\begin{abstract}
The theory of special connectivity [1] has been mathematically verified with the help of probability. With an example of tossing a coin, how consciousness manipulates probability has been discussed. Paper is based on a fact that the observer influences the observed. .

Keywords: Theory of Special Connectivity; Probability; Consciousness; Tossing a Coin; Observer; Observed.

Cite This Article: Prashant. (2018). "GRAVITY, PROBABILITY AND CONSCIOUSNESS." International Journal of Research - Granthaalayah, 6(11), 454-460. https://doi.org/10.29121/granthaalayah.v6.i11.2018.1154.
\end{abstract}

\section{Introduction to Special Connectivity}

According to the theory of special connectivity, whenever an object changes its relative position in gravitational-spacetime, it radiates gravitational waves in the direction of motion and this change is opposed by the effective surrounding gravitational waves. As a result, an infinite web of quadrilateral will be created around the object which is known as the gravity of that object. Object could be anything ranging from an atom to a blackhole $[1,3]$.

Force of gravity of is given by:

$F=\frac{K^{2}}{m a^{3}}$

Where, $\mathrm{K}$ is Kaushal constant of that object, $\mathrm{m}$ is the mass of object and a is the maximum vertical distance from centre of gravity of that object.

Equation 1 can also be rewritten as:

$F=\frac{K_{1} K_{2}}{m a^{3}}$

Where, $K_{1}$ and $K_{2}$ are the Kaushal constants of the objects among which the force of gravity has to be determined, $\mathrm{m}$ is effective mass and $\mathrm{a}$ is the effective radius. If objects are on earth then effective mass and radius will be the mass and radius of the earth. 
Special connectivity also states that if two objects having their individual web of gravitational waves are kept in contact then a new web of gravitational waves will be formed encircling both the individual webs and this new web once formed will always be there even if the two objects are no longer in contact. This new web will always be independent of the individual webs of both the objects [1].

\section{Probability in Terms of Connectivity}

Probability states the likeliness of the occurrence of an event [2]. In Special connectivity, the probability of occurrence reduces for the first iteration and then it becomes 1 for the subsequent iterations. Let us understand this with an example of tossing a coin.

Sample space for tossing a coin is $[\mathrm{H}, \mathrm{T}]$. Hence probability of occurrence is 0.5 . Let us insert some conscious constraints to this sample space. If we take a measurement just before tossing a coin with a constant energy and then observe the outcome, the sample space becomes: [HH, HT, $\mathrm{TH}$, and TT]. Where $\mathrm{HH}$ can be interpreted as, we started with $\mathrm{H}$ and ends up with $\mathrm{H}$ and so on. Hence the new probability of occurrence becomes 0.25 . Tossing a coin with a constant energy for every iteration is a point to be noted.

Suppose we toss a coin with energy $\mathrm{E}$ and got $\mathrm{HH}$ as outcome. Now no matter how many times we toss this coin with same energy $\mathrm{E}$ starting with $\mathrm{H}$, we will always end up with $\mathrm{H}$. Hence for every subsequent iteration, the observer has influenced the observed in such a way that if he starts with $\mathrm{H}$, tosses a coin with same energy $\mathrm{E}$, he will always end up with $\mathrm{H}$ with probability 1 . Let us understand this with the help of theory of special connectivity.

Observer must be having its own web of gravitational waves due to several micromovements throughout his body. Observed i.e. coin must be having its own web of gravitational waves as coin is made up of Fe atoms and every atom has a spin. As soon as observer touches coin, a new web of gravitational waves will be created encircling both the individual webs. This new web can only be stretched but will never get destroyed. With an energy of E, the observer flicks the coin. As soon as the physical contact in between observer and coin breaks, coin is said to be in motion and thus must be making another web of gravitational waves around itself.

Hence coin then must be having two webs of gravitational waves around itself while in physical motion. One due to the motion of atoms inside it and one due to its physical motion Final measurement can only be taken once the coin will came to rest and hence the web due to the physical motion of coin is not of much importance. Observer has now taken the final measurement of outcome and thus making the collective web of observer and observed complete.

Now no matter how many times the observer flicks the coin with energy E, the result will never going to be altered since the result has been decided in the first iteration only and hence the combined web of coin and observer at energy E will never going to be destroyed. Let us do some mathematical manipulations to prove this fact. 


\section{Tossing a Coin}

Let $\mathrm{n}$ be the number to spins the coin will make to cover a distance of $\mathrm{x}$ when it got flicked with energy of E.

Hence, in n number of spins the distance covered is $\mathrm{x}$. So, distance covered in 1 spin is $\frac{x}{n}$.

Since energy of gravitational waves is always quantised [3] and is given by:

$E=K f$

Where $\mathrm{K}$ is the Kaushal constant and is given by: $K=\frac{m G h}{g a^{2}}$ and $\mathrm{f}$ is frequency.

Also, potential energy is given by:

$E=m g h$

Where $\mathrm{m}$ is mass, $\mathrm{g}$ is acceleration due to gravity and $\mathrm{h}$ is height.

On equating 3 and 4 , we get

$h=\frac{K f}{m g}$

Since the distance covered by the coin in

One spin is $\mathrm{x} / \mathrm{n}$. Hence,

$x=\frac{n K_{c} f}{m_{c} g}$

Where $\mathrm{x}$ is the total distance covered, $\mathrm{n}$ is the total number of spins taken, $\mathrm{K}_{\mathrm{c}}$ is Kaushal constant for the coin, $\mathrm{f}$ is frequency, $\mathrm{m}_{\mathrm{c}}$ is the mass of the coin and $\mathrm{g}$ is the acceleration due to gravity.

The energy [1] by which the coin is flicked is given by:

$E=\frac{K_{c}^{2}}{2 m_{c} a_{c}^{2}}$

$\mathrm{K}_{\mathrm{c}}$ is Kaushal constant for the coin, $\mathrm{m}_{\mathrm{c}}$ is the mass of the coin and $\mathrm{a}_{\mathrm{c}}$ is the radius of the coin. Also, kinetic energy of the coin is given by:

$E=\frac{m_{c} v^{2}}{2}$

On equating 7 and 8, we get:

$v=\frac{K_{c}}{m_{c} a_{c}}$ 
Where $\mathrm{v}$ is the velocity of the coin

Since, time $=\frac{\text { distance }}{\text { speed }}$

Hence, using equation 6 and 9, we get:

$t^{2}=\frac{n a_{c}}{g}$

Where it is the time taken by the coin to cover a distance of $\mathrm{x}$ with velocity $\mathrm{v}, \mathrm{n}$ is the total number of spins, $a_{c}$ is the radius of coin and $g$ is the acceleration due to gravity.

\section{Static and Dynamic Web of Gws}

As soon as the coin gets into motion, a new web i.e. dynamic web will be created which is independent of the coin's static web. Static web is there due to the fact that coin is made up of atoms and atoms have a spin.

\subsection{Case One}

When the coin is in observer's hand, a new web will be formed which will be independent of individual webs of the observer and the coin. The energy of the combined web is given by:

$E=E_{o}+E_{c}$

Where $E_{o}$ is the energy of observer's web, given by: $E_{o}=K_{o} f$ and $\mathrm{E}_{\mathrm{c}}$ is the energy of coin's web, given by: $E_{c}=K_{c} f$

Hence, equation 11 becomes:

$E=\left(K_{o}+K_{c}\right) f$

Where $\mathrm{K}_{\mathrm{o}}$ and $\mathrm{K}_{\mathrm{c}}$ are the Kaushal constants of observer and coin respectively.

\subsection{Case Two}

When the observer flicks the coin, one more web of gravitational waves will be created around the coin due to its physical motion.

Hence, total energy of the coin becomes:

$E_{c}=E_{\text {static }}+$ Edynamic

Thus, total energy of combined web becomes:

$E=E_{o}+\left(E_{\text {static }}+E_{\text {dynamic }}\right)$, or

$E=\left(K_{o}+K_{s}+K_{d}\right) f$ 
Where, $\mathrm{K}_{\mathrm{s}}$ and $\mathrm{K}_{\mathrm{d}}$ are the Kaushal constants of the coin, one due to static nature and other due to dynamic nature of the coin.

On comparing equation 12 and 13, we get:

$K_{c}=K_{s}+K_{d}$

\section{Test Data}

Now let us put some test data to verify the above derived equations.

\subsection{Observer}

Mass $\left(\mathrm{m}_{\mathrm{o}}\right)$ of the observer is $77 \mathrm{Kg}$. Maximum vertical distance from centre of gravity $\left(\mathrm{a}_{\mathrm{o}}\right)$ of the observer is $0.66 \mathrm{~m}$.

Hence Kaushal constant $\left(\mathrm{K}_{\mathrm{o}}\right)$ of the observer is $7.988 \times 10^{-43} \mathrm{Kg} \mathrm{m}^{2} \mathrm{~s}^{-1}$.

\subsection{Coin}

Mass $\left(\mathrm{m}_{\mathrm{c}}\right)$ of the coin is $4.95 \times 10^{-3} \mathrm{Kg}$. Radius $\left(\mathrm{a}_{\mathrm{c}}\right)$ of the coin is $1.25 \times 10^{-2} \mathrm{~m}$. Since coin is made up of iron $(\mathrm{Fe})$ atoms, hence, atomic mass of $\mathrm{Fe}$ is $55.845 \mathrm{amu}$ and atomic radius of $\mathrm{Fe}$ is $126 \times 10^{-12} \mathrm{~m}$.

Static Kaushal $\left(\mathrm{K}_{\mathrm{s}}\right)$ of the coin can be calculated as follows:

mass $=9.275 \times 10^{-26} \mathrm{Kg}$ radius $=1.26 \times 10^{-10} \mathrm{~m}$

Hence Kaushal constant of one Fe atom is

$2.64 \times 10^{-50} \mathrm{Kg} \mathrm{m}^{2} \mathrm{~s}^{-1}$

Total number of atoms in mass $\left(\mathrm{m}_{\mathrm{c}}\right)$ of the coin is number of moles $\times N A$, where NA is Avogadro's number. Thus, it becomes $5.338 \times 10^{19}$.

Thus, static Kaushal $\left(\mathrm{K}_{\mathrm{s}}\right)$ of the coin becomes $1.409 \times 10^{-30} \mathrm{Kg} \mathrm{m}^{2} \mathrm{~s}^{-1}$

Dynamic Kaushal $\left(\mathrm{K}_{\mathrm{d}}\right)$ of the coin is $1.432 \times 10^{-43} \mathrm{Kg} \mathrm{m}^{2} \mathrm{~s}^{-1}$

\subsection{Earth}

Since both the observer and the coin are on earth, thus, mass (m) of earth is $5.972 \times 10^{24} \mathrm{Kg}$ and radius (a) of the is $6.371 \times 10^{6} \mathrm{~m}$ 


\section{Verifications}

Let us now apply the test data onto the theory of special connectivity.

\subsection{Force of Gravity}

Force of gravity in between the coin and the observer is: From equation 2,

$F=\frac{K_{o} K_{c}}{m a^{3}}$

Also, from equation 14 ,

$F=\frac{K_{o}\left(K_{S}+K_{d}\right)}{m a^{3}}$

Where $\mathrm{m}$ is the mass of earth and $\mathrm{a}$ is the radius of earth.

Putting values from test data, we get,

$7.2897 \times 10^{-118} \mathrm{~N}$

Since $E=F a$, hence energy becomes,

\section{$4.644 \times 10^{-111} \mathrm{~J}$}

This energy must be equal to the energy of the combined web, from equation 13, we get,

$f=3.295 \times 10^{-81} \mathrm{~Hz}$, or $t=3.034 \times 10^{80} \mathrm{~s}$

Also, time in terms of Kaushal constant [3] is given by

$t=\frac{m a^{2}}{K}$

Since the observer is influencing the observant, hence equation 16 becomes,

$t=\frac{m a^{2}}{K_{o}}$

Putting test data into equation 17 , we get,

$t=3.034 \times 10^{80} \mathrm{~s}$

The above result matches exactly with the equation 15 . Hence it can be concluded that theory of special connectivity is verified. 


\subsection{Probability}

Let us assume that coin has taken only one spin i.e. $\mathrm{n}=1$.

From equation 10, it can be inferred that $t=0.035 \mathrm{~s}$. Since $\mathrm{g}$ is $9.8 \mathrm{~m} / \mathrm{s}^{2}$ and $\mathrm{a}_{\mathrm{c}}$ is the radius of the coin.

We know, Kinetic energy is $\frac{m v^{2}}{2}$ and Potential energy is $m g x$. On equating and solving, we get, $t=\frac{v}{2 g}$. Since $t=0.035 \mathrm{~s}$,

Hence, $v=0.686 \mathrm{~m} / \mathrm{s}$ and thus total distance travelled is $x=2.40 \mathrm{~cm}$. Hence it can again be concluded that theory of special connectivity is verified.

\section{Conclusion}

The observer influences the observed is a point to be noted. In special connectivity, the probability of occurrence reduces for the first iteration and then becomes 1 for all subsequent iterations provided the energy should remain constant. Also, a successful attempt has been made to verify the theory of special connectivity.

\section{References}

[1] Chauhan, P. (2018). Theory of Special Connectivity (Ser. 1). Moldova: Scholars' Press.

[2] Linde, W. (2016). Probability Theory. Doi: 10.1515/9783110466195

[3] Prashant. Kaushal and Gravity. J Phys

[4] Astron. 2018; 6(1):133

*Corresponding author.

E-mail address: rasPhant.941022@gmail.com 